\title{
Critical Flow of Dense Gases - Modeling and Experimental Validation*
}

\author{
J. Górski ${ }^{\mathrm{a} * *}, \mathrm{~S} . \mathrm{Rabczak}^{\mathrm{b}}$ \\ ${ }^{a}$ AGH University of Sciences and Technology, Cracow, Poland \\ jagorski@agh.edu.pl \\ ${ }^{\mathrm{b}}$ Rzeszow University of Technology, Rzeszow, Poland \\ rabczak@prz.edu.pl
}

\begin{abstract}
The critical mass flow of dense gases strongly depends on real gas effects. In the present work the detailed assessment of the critical flow conditions and the limiting mass velocity in the flow of refrigerants has been experimentally verified. Critical flow function $C^{*}$ data for $R-410 A$ and $R-507 A$ have been predicted based on Martin-Hou (MH) equation of the state. The computational study was assured by implementation of a theoretical model (Górski, 1997) for one dimensional (1D) and non-linear gas dynamic problems. This model, with the corrections for the boundary layer (BL) displacement thickness, gives a better prediction of the critical flow function than the classical approach. Appropriate sonic flow conditions have been executed in the pressurized closed loop system by using ISO 9300 critical Venturi nozzle. Measurements of critical mass flow for dense superheated vapour of $R-410 A$ and $R-507 A$ carried out on a laboratory test stand confirmed the accuracy of the model and its physical significance. A main goal of the investigations is a set of charts $C^{*}\left(T_{0}, p_{0}\right)$ and tables developed for an assumed range of stagnation temperature $T_{0}$ and pressure $p_{0}$ in the upstream fluid-flow.
\end{abstract}

Keywords: Refrigerant flow measurements; Critical flow function; Venturi nozzle.

\section{Introduction}

Growing energy consumption associated with local environmental and operating problems of electric, heating and cooling units have led to an increase in the interest in refrigerants not applied for the broader scale so far. Many new devices utilizing low-temperature or waste energy sources in low boiling point applications are being introduced more and more commonly in the various energy conversion systems. The most useful agents for these applications are the organic fluids as propane, butane and inorganic substances (carbon dioxide), and a whole range of ozone depletion free CFC replacements.

Detailed data referring to the estimation of their critical flow characteristics are not available for many of them, particularly in the high-pressure area (used e.g. in the transcritical cycles). The implementation of an ideal gas model to the process calculations in a real compressible fluid can lead to large discrepancies between measured and predicted values of state parameters. Currently, very noticeable is the development of methods for applying the fundamental equations of state (EOS's) not only within applied process thermodynamics but also in the numerical fluid flow analysis (Thompson, Sullivan, 1977).

At the principal study and qualitative examination of any physical processes the most useful and still popular is a simple van der Waals (vdW) model. A main weakness of such approach is a poor quantitative level of the obtained particular numerical results. More sophisticated methods are involving the application of multi-parameter EOS's and special computer routines for the calculation of the necessary parameters. In an engineering practice the most popular and well known equations of state are the Martin-
Hou (MH) and the generalised modified Benedict-WebbRubin (MBWR) EOS (Span, 2000). These EOS's have been widely applied and actually used for pure new refrigerants and refrigerant blends, for example $R-134 a, R$ $407 C, R-410 A$ and $R-507 A$.

The main purpose of this work is to deliver useful tools and data for the prediction of the critical (sonic) conditions in a compressible and high-density flow of gases or vapours. Based on the source data (Annon, DuPont) for near azeotropic blend refrigerant $R-410 A$ and the zeotropic blend $R-507 A$, an experimental verification of the theoretical results were performed.

In the present work authors propose an effective tool for the prediction of the critical flow of vapour-phase refrigerants and high-density technical gases proving its usefulness and correctness on the basis of experimental results.

\section{Research Problem}

A critical or sonic nozzle is a device to measure the mass flow with only the nozzle supply conditions making use of flow choking phenomenon at the throat (ISO 9300:2005). An accurate prediction of the mass flow rate and the critical flow function is of practical importance since the mass flow rate is essentially associated with limiting the working gas consumption or the accidental release flow rates from pressurized gas systems. Critical pressure ratio should be known to establish the operating conditions for safety and design the expansion valves in the high-pressure refrigeration units and Organic Rankine Cycle (ORC) systems, measuring natural gas flows and many other applications (Johnson, 1971; Annon, 1997). 
According to classical 1D gas dynamics, the mass flow rate is a function of the pressure and temperature at upstream stagnation conditions, the diameter of nozzle throat and specific heats ratio. This phenomenon is one of the unique features of compressible internal flows. In compressible flow with a sufficiently high backpressure ratio, the flow is choked at minimum cross section $A_{*}$ and the mass flow rate reaches its maximum value. At the critical pressure ratio the flow is no longer dependent on pressure changes downstream and flow the mass flow is determined only by fluid upstream stagnation conditions.

In the one-dimensional (1D) steady isentropic flow $\left(s_{0}=s_{*}\right.$ = idem.) an energy equation is simply given by

$h_{0}=h+\frac{w^{2}}{2}=h_{*}+\frac{a_{*}^{2}}{2}=i d e m$.

For the sonic flow conditions at Mach Number $M a=1$, the flow velocity equals to the sound speed $w=a_{*}$, and the static parameters correspond to the critical ones $\left(T=T^{*}, p\right.$ $\left.=p^{*}, h=h^{*}, a=a^{*}\right)$. All complete relations in a compressible critical flow of the perfect gases can be found anywhere in the lectures on classical gas dynamics. The critical mass flow $\dot{m}_{*}$, and related critical flow functions are respectively defined

$$
\begin{aligned}
& \dot{m}_{*}=C^{*} \cdot \frac{A_{*} p_{0}}{\sqrt{R T_{0}}}, \\
& C^{*}=\frac{\dot{m}_{*} \sqrt{R T_{0}}}{A_{*} p_{0}}, \text { or } \Psi^{*}=\frac{\dot{m}_{*}}{A_{*} \sqrt{p_{0} \rho_{0}}}=\sqrt{\frac{C^{*}}{z}} .
\end{aligned}
$$

The calculated theoretical values may differ from the experimental data and an actual mass flow $\dot{m}_{*}^{\exp }$ should be corrected. Considering the well-known discharge coefficient $C_{d}$, it is possible to account for the three dimensional (3D) effects $C_{3 D}$, non-isentropic process $C_{\gamma}$ and viscous (boundary-layer) phenomena $C_{B L}$, see Johnson (2000)

$$
C_{d}=\dot{m}_{*}^{\exp } / \dot{m}_{*}, \quad \text { and } \quad C_{d}=C_{3 D} \cdot C_{\gamma} \cdot C_{B L}
$$

For sufficiently high Reynolds numbers and the steady uniform flow, the discharge coefficient approaches unity, indicating that the 1D inviscid model is valid for prediction of the maximum mass flow. For low Reynolds numbers the discharge coefficient reduces considerably below unity. This is due to the boundary layer growth in the flow through the sonic nozzle. For a small critical nozzle, the Reynolds number can be low, and in this case the prediction of the mass flow rate is not straightforward since it depends on downstream pressure variations, even under the condition of the critical pressure ratio.

\section{Critical Isentropic Flow}

According to ideal gas dynamics, in the steady 1D flow, the flow rate is a function of the pressure ratio and of the gas properties. The problem of the critical flow peculiarities in the dense gases was previously treated by many authors (Leung, Epstein 1988; Bober, Chow, 1977; Shumann, 1990). For CFC refrigerants $R-12, R-22$ and $R$ -
502 , the theoretical analysis was presented by Shumann (1990). Górski (1997) proposed in his monograph a unified approach for the analysis of the thermal-flow processes for an arbitrary real gas model. The method of the Virial Compressibility Derivatives (VCD) gives more clear results which can be directly related to an ideal gas model.

In the case of 1D dense gas steady flow, the wellknown relations presented in classical books on gas dynamics are in general invalid. It is caused by strong variation of physical constants and all thermal and caloric properties involved in the calculation of the process. As is shown (Górski, 1997), it is possible to find the analogous parameters to the isentropic flow of real gases, similar to an ideal gas flow. The approximate relations between critical and stagnation parameters in the sonic flow can be shortly expressed as (Górski, 1997)

$\frac{T^{*}}{T_{0}} \cong\left(\frac{2}{\chi+1}\right)^{\frac{\phi}{\chi-1}}, \frac{\rho^{*}}{\rho_{0}} \cong\left(\frac{2}{\chi+1}\right)^{\frac{1}{\chi-1}}$,

$\frac{p^{*}}{p_{0}}=\beta^{*} \cong\left(\frac{2}{\chi+1}\right)^{\frac{k}{\chi-1}}, \quad$ where

$\Phi=\frac{\rho}{T}\left(\frac{\partial T}{\partial \rho}\right)_{s}=\frac{z_{T}}{\bar{c}_{v}}, k=\frac{\rho}{p}\left(\frac{\partial p}{\partial \rho}\right)_{s}=\gamma \frac{z_{v}}{z}$,

$\chi=k+\frac{\rho}{k}\left(\frac{\partial k}{\partial \rho}\right)_{s}=2 \Gamma-1, \quad$ and

$z=\frac{p}{\rho R T}, z_{v}=z-v\left(\frac{\partial z}{\partial v}\right)_{T}=z+\rho\left(\frac{\partial z}{\partial \rho}\right)_{T}$,

$z_{T}=z+T\left(\frac{\partial z}{\partial T}\right)_{v}, \gamma=\frac{c_{p}}{c_{v}}, \bar{c}_{v}=\frac{c_{v}}{R}$.

In Eqns (4) and (5) the Grüneisen parameter $\Phi$ and the generalized isentropic exponent $\chi$ (corresponding to the fundamental derivative $\Gamma$ ) have been introduced, see Thompson \& Sullivan (1977) and Shumann (1990). Other important local factors are: the specific heat ratio $\gamma$, isentropic exponent $k$, compressibility factor and the VCD derivatives $z_{T}$ and $z_{v}$. These parameters can be simply found from the thermal EOS usually given by $p=p(T, v)$. For a perfect gas case, all factors in Eqns (4) - (6) take the values of well-known constants

$z=z_{T}=z_{v}=1, \chi=k=\gamma, \Phi=R / c_{v}$,

as well as the critical flow function and ratio of local sonic state parameters would only depend on the specific heat ratio. In a real gas flow all the above mentioned parameters are functions of stagnation temperature and pressure, see the reciprocal of $\beta_{*} \equiv 1 / \beta_{k r}$, presented in the Figure 1 .

The calculation results of the critical pressure ratio in an isentropic flow of real gases for refrigerant $R-410 A$ show not only an influence of the stagnation temperature but the strong effect of the stagnation pressure variation.

Assuming the isentropic exponent $k=1.125$ for $\mathrm{R}$ $410 \mathrm{~A}$ at the normal conditions, in an ideal gas case the corresponding value of $p_{0} / p^{*}=1 / \beta^{*}=1.726$. From Figure 1 , one can easy see that this parameter can vary between 1.67 and 1.75, and an even wider range is closer to the saturation line $(x=1)$. 


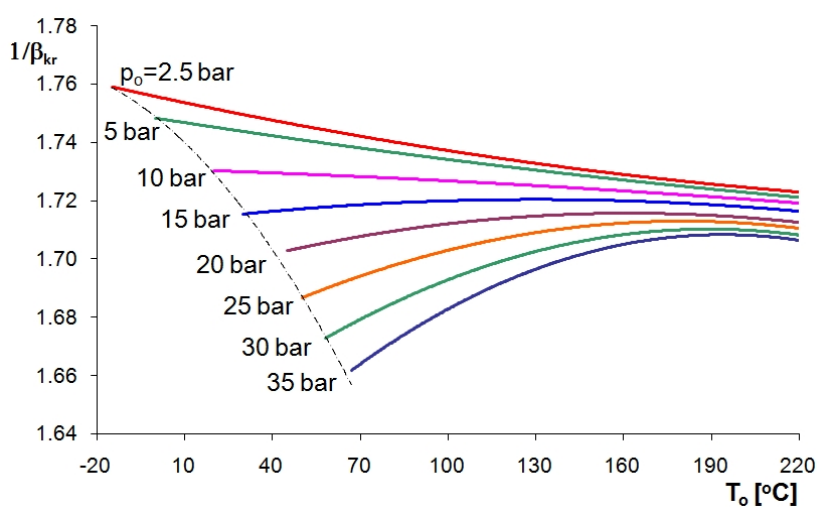

Figure 1. Critical pressure ratio for vapour of $R-410 A$.

Using Martin-Hou EOS and the Eqns (1) - (6), the critical flow factor $C^{*}$ was calculated for dense refrigerant vapour over the range 300 to $500 \mathrm{~K}$ and 0 to $3.5 \mathrm{MPa}$ (excluding the area where the condensation occurs) for two refrigerant blends $R-410 A$ and $R-507 A$. The calculation procedures are iterative but fast convergent. They start with the initially estimated flow conditions at the throat, i.e. temperature, pressure and density. It can be found from relations (4) for the estimated values of coefficients (5). In the next iteration step, all data corresponding to the mean values of parameters accounted from (5), allow to calculate and obtain new results much closer to the final ones. The end of the iterative procedure is only limited by the assumed and acceptable discrepancies for the enthalpy and entropy equalities given by Eqn. (1).

\section{Experimental Tests}

A main goal of this work was an experimental verification of theoretical results and to compare data from laboratory tests to the computational ones. Rabczak (2007) developed an original test-bench in order to attain the sonic conditions in a closed refrigeration circuit. This facility is operating based on the Critical Venturi Nozzles (CVN). Superheated vapours of refrigerants $R-410 A$ and $R-507 A$ were used as working media. Based on the computational study the selection of two ISO-9300 Venturi nozzles (ISO 9300:2005), with the throat diameter $d=0.8$ (only for $R$ $507 A$ ) and $1.0 \mathrm{~mm}$ (both refrigerants) were made. The critical nozzle of $0.8 \mathrm{~mm}$ diameter was used for the upper range of stagnation pressures $p_{0}>1.5 \mathrm{MPa}$. At the smaller pressures, a $1.0 \mathrm{~mm}$ throat nozzle was taken in order to preserve the heat capacity of the continuous-flow calorimeter. A steady-state flow calorimeter method was applied for selective measuring of the thermal and flow parameters of refrigerant blends. This calorimetric method ensures precise and stable measurement conditions in the main circuit.

The principal schema of the research meter circuit and instrumentation is shown in the Figure 2. Circulating tested refrigerant, in the main measuring line (high pressure vapour), flows between two pressure vessels: high DCR0487s Danfoss (1), and low pressure - DCR0967s (2). In this pipeline the critical Venturi nozzle (9), thermocouples (T1, T2 - type Pt100) and the pressure gauges (P1, P2 - type AKS Danfoss) were installed. Vessels (1) and (2) serve as the pressure buffers, whereas the required conditions for the demanded stagnation temperature and pressure level in the installation were assured by two compressors ML80TB (7), water cooler HE1.0 Danfoss (3), 2.1 sq. m condenser (6), $1 \mathrm{~kW}$ electric heater (10) and control valves. For keeping the suitable pressure in the system, a pressostatic expansion valve $P Z$ Fach (13) was operating on the high pressure side. Fluid conditions on the low-pressure side (liquid refrigerant) have been forced by $1 \mathrm{~kW}$ flow calorimeter PP51 (class $0.5)$ with $R-134 a$ as the secondary fluid (5). Three calibrated rotameters DK37E Krohne (4), installed on the liquid side of cooling circuit, assure the control of the fluid mass flow and maintain the energy balance of the flow calorimeter. The stable conditions required for the measurements were obtained after 3 to 4 hours. The uncertainty of the temperature close to the critical nozzle (T1/T2 gauges) was $\pm 0.1 \mathrm{~K}$, and the pressure $\pm 0.5 \%$ (P1/P2 gauges, see Figure 2). Less important temperature and pressure data in the remaining points were measured with a smaller accuracy $( \pm 0.3 \mathrm{~K}$ and $\pm 1 \%$ respectively). The operating conditions of the proposed test loop were limited up to $p_{0} \leq 35$ bar and $T_{0} \leq 110{ }^{0} \mathrm{C}$ behind the refrigerant saturation line in order to avoid two-phase flow in the main pipeline. The equipment components and gauges used for the measurements allowed to obtain a maximum uncertainty level below $1.7 \%$. During test-bench operation the data were collected in the computer storage with the

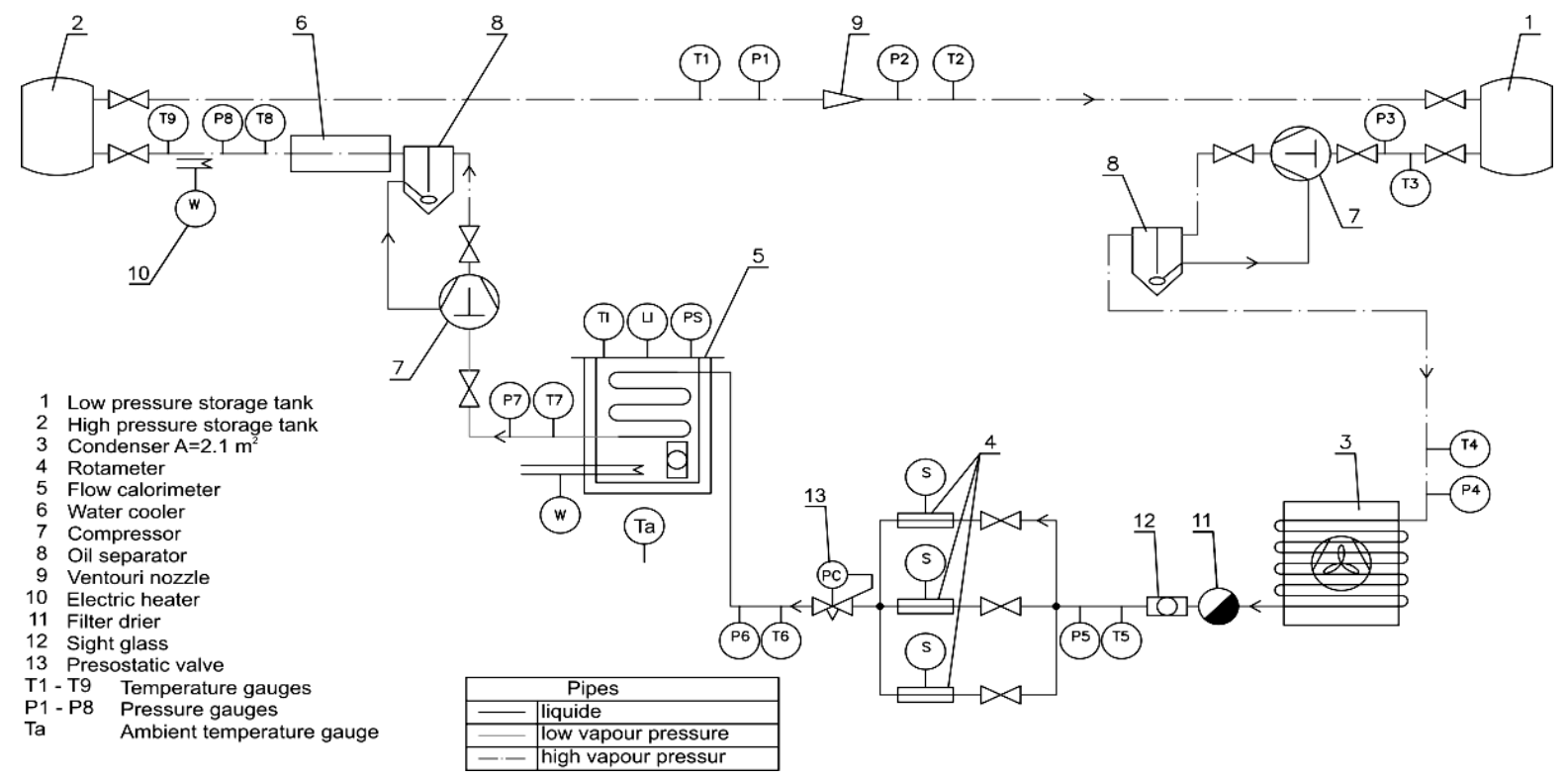

Fig. 2. Schema of test - bench for measurement of critical flow function,(Rabczak, 2007). 
sequence of 1 second interval. Stabilized conditions in the circuit have been reached after about 4 to 5 hours, and the data were collected to obtain the critical mass flow and critical flow function. The operating conditions of the test loop were limited up to $p_{0} \leq 3.5 \mathrm{MPa}$ and $T_{0} \leq 110^{\circ} \mathrm{C}$ in superheated vapour area in order to avoid two-phase flows in the main circuit. The principal view of the circuit loop is presented in the Figure 3. All of the main components have been coated by the polyethylene foam assuring thermal insulation from the surrounding.

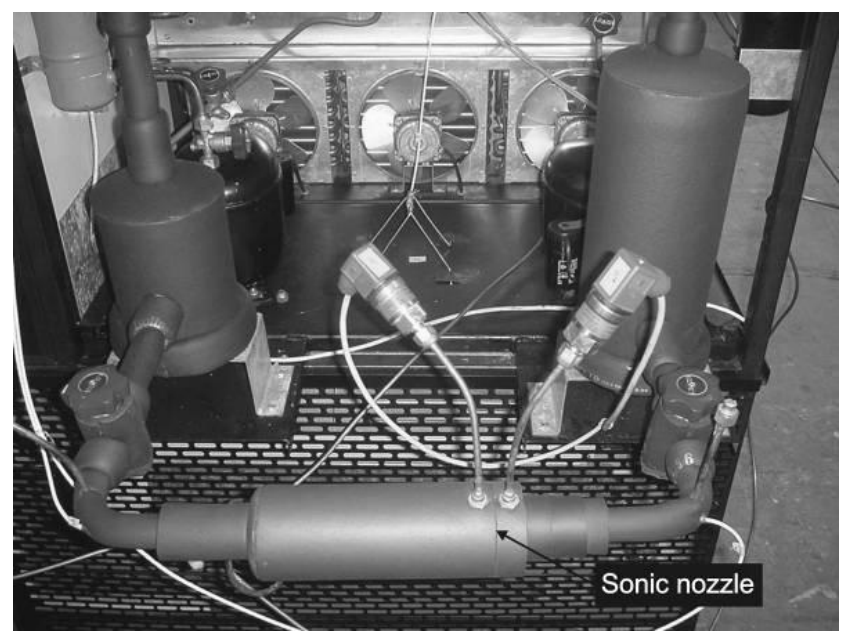

Figure 3. View on the sonic nozzle on test bench,(Rabczak, 2007).

\section{Results and Discussion}

In the experimental part of this investigation all data on the critical function $C^{*}$ for $R-410 A$ and $R-507 A$ at the selected 24 test points have been obtained and compared to the previously predicted theoretical results.

Figure 4 presents a critical flow function for superheated vapour of $R-410 A$ refrigerant comparing experimental data the theoretical model results. Obtained experimental data are in a close agreement with computations analysis results based on the $\mathrm{MH}$ equation of state.

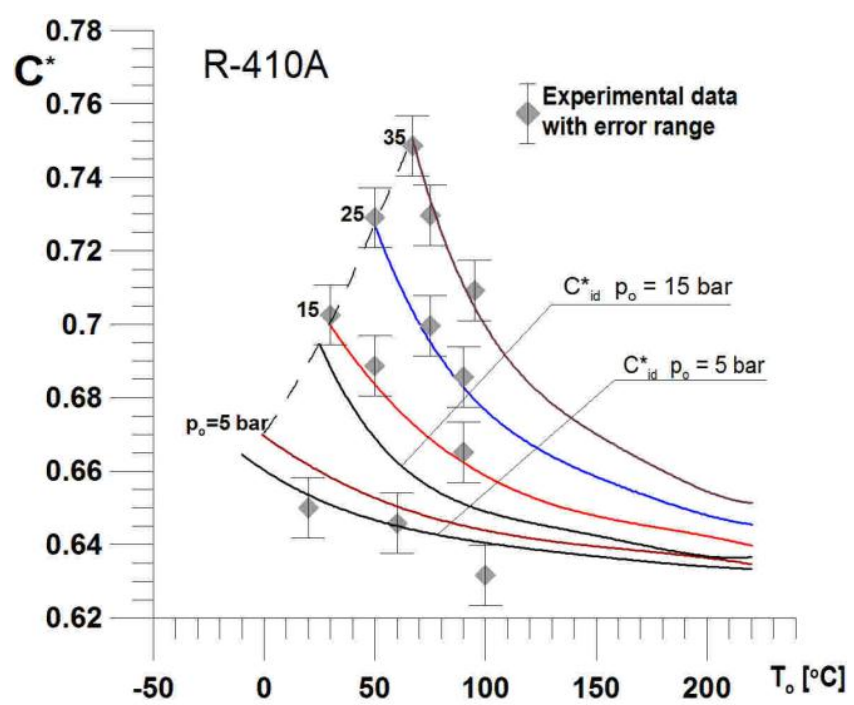

Figure 4. Critical flow function vs. stagnation parameters theoretical and experimental data for R-410A (Rabczak, 2007).
The average discrepancy between theoretical and experimental results is less then $1.0 \%$. It confirms that the new analytical model is valid and formulated in accordance to the real physical phenomena. The experimental data up to pressure 5 bars are in better correspondence with ideal gas behavior, therefore these results of dense gas flow analysis are applicable to the high pressure region and higher than 5 bars.

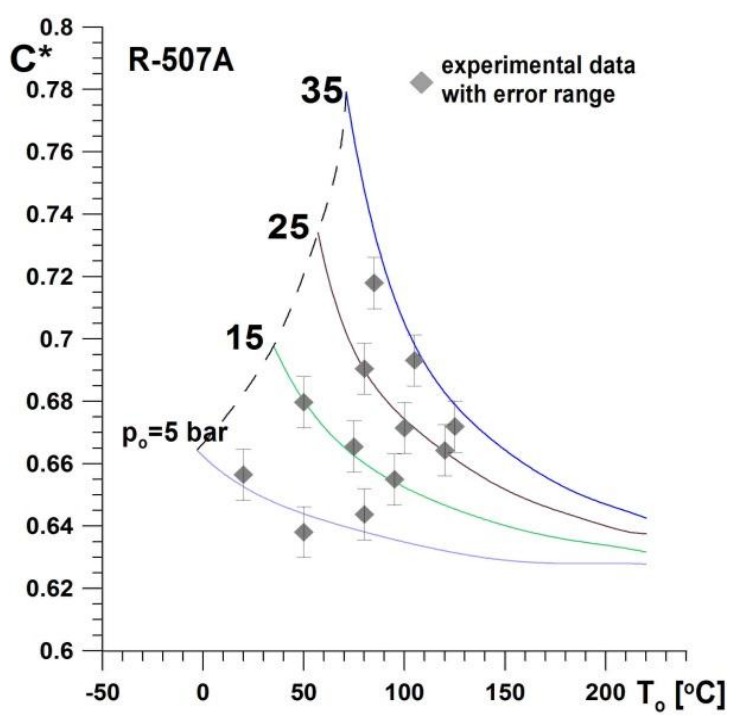

Figure 5. Critical flow function vs. stagnation parameters theoretical and experimental data, R-507A, (Rabczak, 2007).

All experimental points in the Figure 4 and 5 take into consideration the boundary layers corrections $C_{\delta}$ and as well as speed distribution $C_{K}$ and viscosity effect $C_{\gamma}$ corrections. Total correction factor $C_{d}$, is calculated according to the equation (Johnson, 2000)

$C_{d}=C_{K} \cdot C_{\delta} \cdot C_{\gamma}$

Based on that formulated correction terms the real critical flow factor $C^{*}$, was obtain by the ratio of experimental value $C_{\text {exp }}^{*}$ to the above given factor $C_{d}$

$C^{*}=C_{\exp }^{*} / C_{d}$

The boundary layers correction factor $C_{\delta}$ proposed by Geropp \& Tang (1971) takes the form

$$
C_{\delta}=1-\left(\frac{\gamma+1}{2}\right)^{1 / 4}\left[\frac{8(9-4 \sqrt{6})}{3(\gamma+1)}+\frac{4 \sqrt{6}}{3}\right] \cdot\left(\frac{d}{2 R_{c}}\right)^{-1 / 4} \frac{1}{\sqrt{R e}} \cdot
$$

This correction term due to boundary layer (BL) presence is applicable for the Reynolds Numbers $R e>2000$. It is possible to calculate the BL displacement thickness $\delta$, by using the mentioned Geropp's (1971) model and an assumed approximate relation

$$
\frac{2 \delta^{*}}{d}=0.001309+1.72169 \frac{1}{\sqrt{\operatorname{Re}}} .
$$


Figure 6 shows the correlation between BL thickness and the Venturi nozzle throat $d$ in the range corresponding to the experiment conditions $\left(\operatorname{Re}=2 \cdot 10^{5}\right.$ to $\left.1.2 \cdot 10^{5}\right)$. As one can notice in all analyzed cases the flow is fully developed and turbulent.

At the low flow velocities the boundary layer thickness strongly increases for $R e<6 \cdot 10^{5}$. At the higher Reynolds Numbers, the BL displacement thickness for the selected two nozzles are in a narrow range. The replacement of the nozzle throat diameter from 0.8 to $1.0 \mathrm{~mm}$ causes only an approximately $0.58 \mu \mathrm{m}$ change in the BL displacementthickness. Comparable results were obtained by Johnson (1971; 2000) and presented in the report AR-321 (1997).

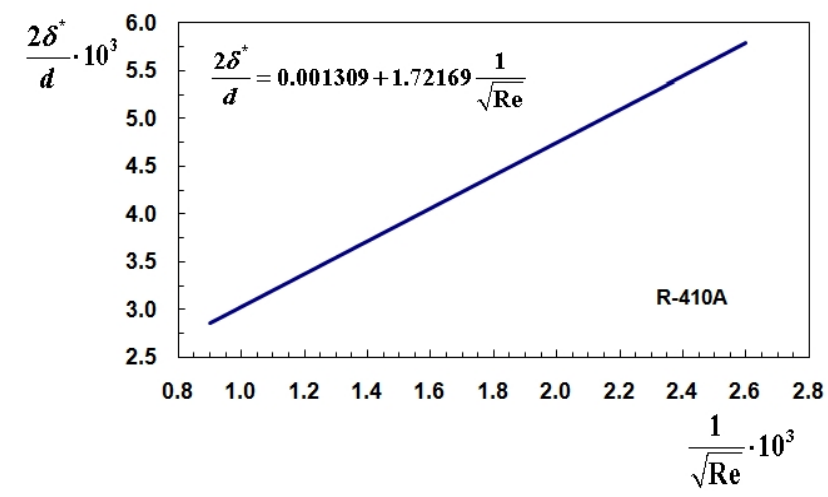

Figure 6. Relative boundary-layer displacement thickness vs. Reynolds Number, (Rabczak, 2007).

If one looks at boundary layer correction factor $C_{\delta}$, it depends on type of flow and geometric dimension of used critical Venturi nozzles in accordance to Figure 7.

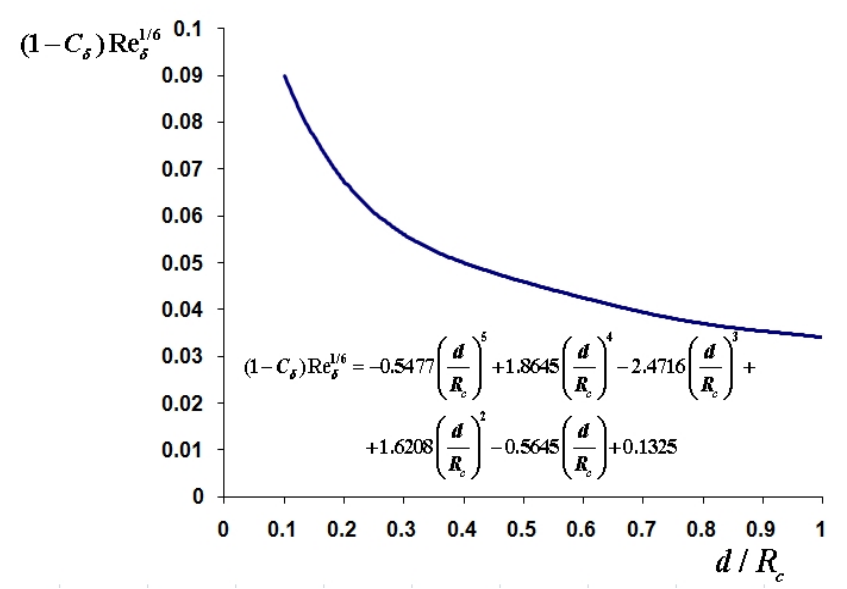

Figure 7. Boundary-layer correction factor $C_{\delta} v s$. dimensions of Venturi nozzle, (Rabczak, 2007).

Other obtained results of viscosity correction factor $C_{\gamma}$ for $R-410 A$ are presented in Figures 8-9.

The most well-known formula for the real gas effect correction factor $C_{\gamma}$ was proposed by Johnson (2000)

$C_{\gamma} \approx \sqrt{\gamma_{o}}\left(\frac{\gamma_{o}+1}{2}\right)^{\frac{\gamma_{o}+1}{2\left(1-\gamma_{o}\right)}}$.

In order to account the velocity distribution correction factor the suitable formula was delivered by Hall (in Johnson, 2000). A value of this correction factor $C_{K}$ has been presented in Figure 9, expanded in view to geometric dimension of measurement Venturi nozzle, i.e. throat diameter $d$ to the curvature radius $R_{c}$ ratio.

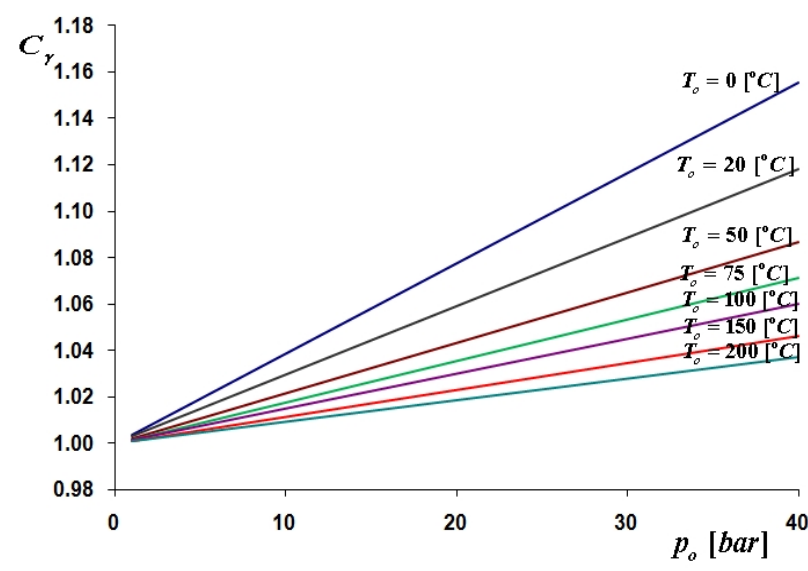

Figure 8. Viscosity effect correction factor $C_{\gamma}$ vs. stagnation pressure, (Rabczak, 2007).

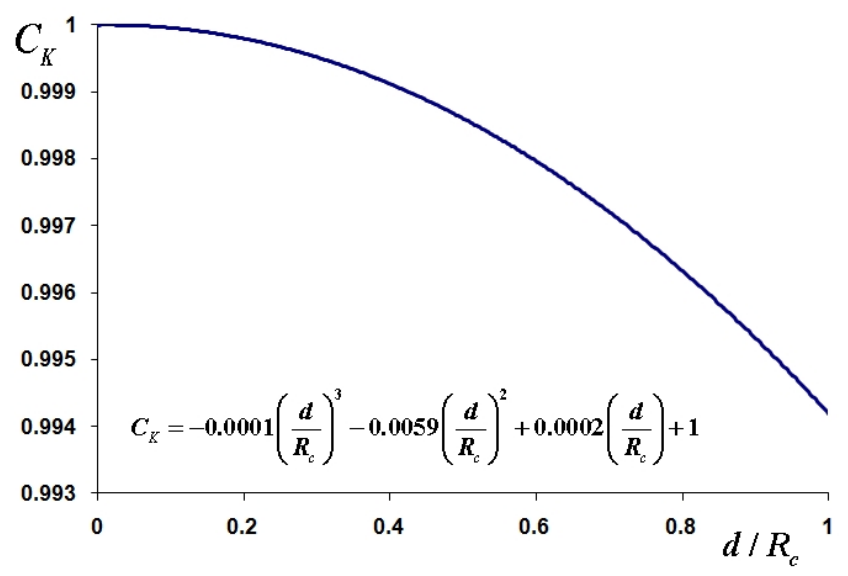

Figure 9. Velocity distribution correction factor $C_{K}$ vs. non-dimensional throat diameter, (Rabczak, 2007).

Assuming the thermal conditions of temperature as $T_{o}=50{ }^{\circ} \mathrm{C}$ and pressure $p_{o}=25$ bars for $R-410 A$, the important parameters found from theoretical compressible fluid model are presented in the Table 1.

Table 1. Selected parameters related to calculation of $C_{d}$ correction factor for $R-410 A$.

\begin{tabular}{|c|c|c|c|c|c|c|}
\hline$a_{s}$ & $s_{o}$ & $C^{*}$ & $k_{s}$ & $C^{i d}$ & $R e \cdot 10^{5}$ & $\gamma_{o}$ \\
\hline$[\mathrm{m} / \mathrm{s}]$ & {$[\mathrm{J} / \mathrm{kgK}]$} & {$[-]$} & {$[-]$} & {$[-]$} & {$[-]$} & {$[-]$} \\
\hline \multicolumn{7}{|c|}{$\left(T_{o}=50{ }^{\circ} \mathrm{C} ; p_{o}=25\right.$ bars $)$} \\
\hline 165.32 & 1784.6 & 0.7271 & 1.052 & 0.7095 & 7.54 & 1.22 \\
\hline
\end{tabular}

For a principal nozzle dimension $d=1.0 \mathrm{~mm}( \pm 10 \mu \mathrm{m})$ and inlet radius $R_{c}=0.8 \mathrm{~mm}( \pm 10 \mu \mathrm{m})$, all the above correction factors are respectively equal to

$$
\begin{aligned}
& C_{K}=0.9942, \\
& C_{\delta}=0.9960, \\
& C_{\gamma}=1.0631 .
\end{aligned}
$$

Ultimately, the discharge factor for $R-410 A$ refrigerant in this analyzed point is directly accounted as 
$C_{d}=0.9942 * 0.9960 * 1.0631=1.0527$.

A value of the flow factor for the stagnation conditions at the experimental conditions equals to $C_{\text {exp }}^{*}=0.69252$.

The real gas critical flow factor is simply found as ratio of $C_{\text {exp }}^{*}$ and correction factor $C_{d}$, thus

$C^{*}=0.69252 / 1.0527=0.7289$.

Compared to the value from mathematical model $C^{*}=$ 0.7271 it is less than $0.3 \%$ of the real value relative error. The obtained theoretical values of critical flow factor $C^{*}$ for $R-507 A$ are presented in the Table 2. These data include a wide range of stagnation parameters, i.e. temperature ( 240 to $490 \mathrm{~K}$ ) and pressure ( 1 to 35 bars). The blank parts in this table are corresponding to the two-phase flow regime. The large variations in the $C^{*}$ function is easily observed close to the saturation line. The temperature rise leads to the magnitude decreasing in fluctuation of critical flow factor.

Uncertainties have been estimated for the experimental and theoretical values of the flow function $C^{*}$ and mass stream in an analysis of 1D critical flow. The standard error of measurement (SEM) was estimated for two analysed fluids and the obtained data have been referred to the experimental ones and bias limits. In the case of an ideal gas model the relative error of function $C^{*}$ is within 0.5 to $2.5 \%$ for $R-507 A$, but from the $\mathrm{MH}$ real gas model not exceeded 0.3 to $0.9 \%$ (only one point has been failed outside the range), see Figure 10. All results are compared for the certain stagnation temperature range ( 85 to 125,80 to 120,50 to 95 and 20 to $80^{\circ} \mathrm{C}$ ) and the stagnation pressure conditions $(35,25,15$ and 5 bars) respectively.

\section{Conclusions}

The paper compares new results of computational analysis of dense gas critical mass flow to experimental data. The validity of analytical tools presented in the work of Górski (1997) has been confirmed by research tests in the PhD thesis (Rabczak, 2007). The final results of this work are summarized as follows:

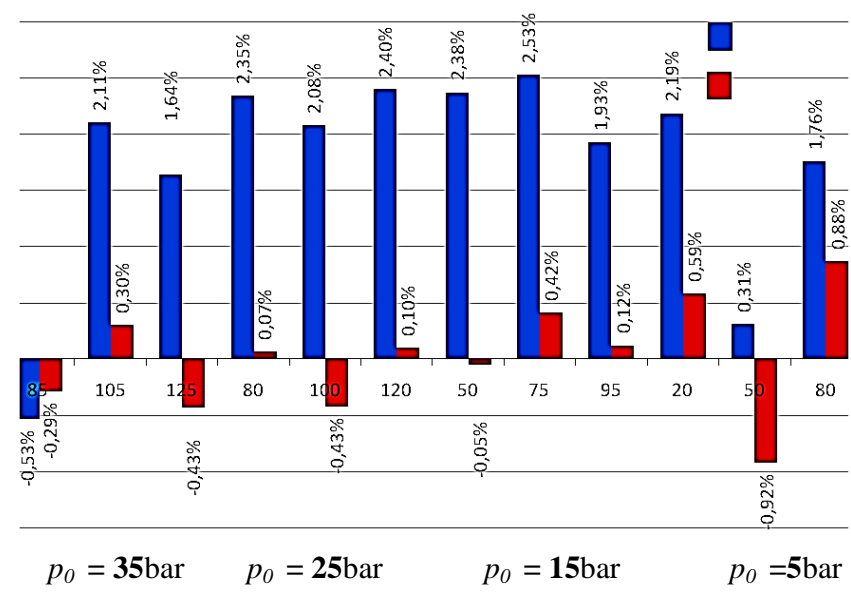

Figure 10. Relative uncertainty of critical flow function $C^{*}$ for $R$ 507A (red bars - experiment, blue - ideal gas), (Rabczak, 2007).

Table 2. Critical flow factor for R-507A superheated vapour, (Rabczak, 2007).

\begin{tabular}{|c|c|c|c|c|c|c|c|c|c|}
\hline \multirow{2}{*}{$\begin{array}{c}C^{*} \\
T_{o}[\mathrm{~K}]\end{array}$} & \multicolumn{9}{|c|}{$p_{o}[\mathrm{bar}]$} \\
\hline & 1 & 2,5 & 5 & 10 & 15 & 20 & 25 & 30 & 35 \\
\hline 240 & 0.6437 & & & & & & & & \\
\hline 250 & 0.6419 & 0.6518 & & & & & & & \\
\hline 260 & 0.6402 & 0.6493 & & & & & & & \\
\hline 270 & 0.6386 & 0.6469 & 0.6612 & & & & & & \\
\hline 280 & 0.6371 & 0.6447 & 0.6577 & & & & & & \\
\hline 290 & 0.6357 & 0.6426 & 0.6544 & 0.6770 & & & & & \\
\hline 300 & 0.6344 & 0.6406 & 0.6513 & 0.6701 & & & & & \\
\hline 310 & 0.6331 & 0.6388 & 0.6483 & 0.6642 & 0.6952 & & & & \\
\hline 320 & 0.6319 & 0.6370 & 0.6456 & 0.6592 & 0.6833 & 0.7153 & & & \\
\hline 330 & 0.6308 & 0.6354 & 0.6430 & 0.6549 & 0.6742 & 0.6981 & & & \\
\hline 340 & 0.6299 & 0.6339 & 0.6406 & 0.6513 & 0.6672 & 0.6856 & 0.7094 & & \\
\hline 350 & 0.6289 & 0.6326 & 0.6384 & 0.6481 & 0.6617 & 0.6764 & 0.6937 & 0.7222 & 0.7578 \\
\hline 360 & 0.6281 & 0.6313 & 0.6364 & 0.6454 & 0.6572 & 0.6695 & 0.6835 & 0.7041 & 0.7298 \\
\hline 370 & 0.6274 & 0.6302 & 0.6346 & 0.6431 & 0.6535 & 0.6641 & 0.6762 & 0.6918 & 0.7100 \\
\hline 380 & 0.6267 & 0.6292 & 0.6330 & 0.6410 & 0.6503 & 0.6596 & 0.6704 & 0.6828 & 0.6959 \\
\hline 390 & 0.6261 & 0.6283 & 0.6316 & 0.6391 & 0.6475 & 0.6558 & 0.6653 & 0.6755 & 0.6855 \\
\hline 400 & 0.6257 & 0.6275 & 0.6303 & 0.6375 & 0.6450 & 0.6524 & 0.6607 & 0.6692 & 0.6777 \\
\hline 410 & 0.6253 & 0.6269 & 0.6293 & 0.6360 & 0.6428 & 0.6493 & 0.6565 & 0.6638 & 0.6713 \\
\hline 420 & 0.6249 & 0.6264 & 0.6284 & 0.6346 & 0.6407 & 0.6466 & 0.6528 & 0.6593 & 0.6659 \\
\hline 430 & 0.6247 & 0.6260 & 0.6278 & 0.6333 & 0.6390 & 0.6443 & 0.6497 & 0.6556 & 0.6612 \\
\hline 440 & 0.6246 & 0.6257 & 0.6273 & 0.6322 & 0.6374 & 0.6422 & 0.6470 & 0.6526 & 0.6570 \\
\hline 450 & 0.6245 & 0.6256 & 0.6270 & 0.6311 & 0.6361 & 0.6406 & 0.6448 & 0.6501 & 0.6534 \\
\hline 460 & 0.6245 & 0.6256 & 0.6269 & 0.6303 & 0.6351 & 0.6391 & 0.6427 & 0.6477 & 0.6503 \\
\hline 470 & 0.6246 & 0.6257 & 0.6270 & 0.6297 & 0.6341 & 0.6379 & 0.6406 & 0.6452 & 0.6477 \\
\hline 480 & 0.6248 & 0.6259 & 0.6273 & 0.6293 & 0.6332 & 0.6366 & 0.6389 & 0.6426 & 0.6455 \\
\hline 490 & 0.6251 & 0.6262 & 0.6278 & 0.6292 & 0.6321 & 0.6351 & 0.6380 & 0.6406 & 0.6433 \\
\hline
\end{tabular}


- The critical flow function for dense refrigerants strongly depends on the upstream stagnation conditions.

- The computation results for $R-410 A$ and $R-507 A$ are in satisfactory agreement with experimental data.

- The proposed approximation of critical flow conditions for real dense gases is better than other comparable methods, see Standard ISO 9300:2005 and AGARD-AR321 (1997).

- At the stagnation pressure exceeding $p_{0}>0.5 \mathrm{MPa}$, the perfect gas model is not applicable in the flow of dense and superheated vapour of refrigerants.

- The boundary layer (BL) at the sonic nozzle throat is turbulent and affects the critical flow function.

- Other theoretical results have been actually developed for $R-22, \quad R-123, \quad R-125, \quad R-134 a, \quad R-143 a, \quad R-152 a$, $R-227, R-404 A, R-407 C, R-410 A, R-507 A$ and $C_{2}$. The measurements of the critical flow conditions and its peculiarities will be finished and published soon.

- The future work will aim to develop more universal correlations for determining the critical flow conditions and flow function $C^{*}$ for similar fluids. It will be done based on generalized corresponding states correlations and extended similarity principles, see Leung \& Epstein (1988).

\section{Acknowledgements}

Authors gratefully acknowledge the financial support provided by the funding supported in part by the State Committee for Scientific Research of the Republic of Poland (KBN) under Research Grant 4 T07A 04629 and Dr W. Warczak from COCh Krakow, for his help in the development of experimental stand.

\begin{tabular}{|c|c|}
\hline \multicolumn{2}{|c|}{ Nomenclature } \\
\hline$A^{*}$ & critical throat area $\left[\mathrm{m}^{2}\right]$ \\
\hline$a$ & speed of sound $[\mathrm{m} / \mathrm{s}]$ \\
\hline$C^{*}$ & critical flow function [-] \\
\hline$C_{d}$ & discharge coefficient, correction factor [-] \\
\hline$c_{p}, c_{v}$ & specific heats $[\mathrm{J} / \mathrm{kg} \mathrm{K}]$ \\
\hline$d$ & nozzle throat diameter $[\mathrm{mm}]$ \\
\hline$h$ & specific enthalpy $[\mathrm{kJ} / \mathrm{kg} \mathrm{K}]$ \\
\hline$k$ & isentropic exponent [-] \\
\hline$M a$ & Mach Number [-] \\
\hline$\dot{m}$ & mass flow [kg/s] \\
\hline$p$ & pressure $[\mathrm{Pa}]$ \\
\hline$R$ & gas constants $[\mathrm{J} / \mathrm{kg} \mathrm{K}]$ \\
\hline$R_{c}$ & inlet nozzle radius $[\mathrm{m}]$ \\
\hline $\operatorname{Re}$ & Reynolds Number [-] \\
\hline$s$ & specific entropy $[\mathrm{J} / \mathrm{kgK}]$ \\
\hline$T$ & temperature $[\mathrm{K}]$ \\
\hline$v$ & specific volume $\left[\mathrm{m}^{3} / \mathrm{kg}\right]$ \\
\hline$w$ & gas velocity $[\mathrm{m} / \mathrm{s}]$ \\
\hline$z$ & compressibility factor $[-]$ \\
\hline$z_{T}, z_{v}$ & VCD's, equation (6) [-] \\
\hline$\beta^{*}$ & critical pressure ratio [-] \\
\hline$\Gamma$ & fundamental derivative, equation (5) [-] \\
\hline$\gamma$ & specific heats ratio $[-]$ \\
\hline$\delta^{*}$ & BL displacement-thickness [mm] \\
\hline
\end{tabular}

$\begin{array}{ll}\rho & \text { density }\left[\mathrm{kg} / \mathrm{m}^{3}\right] \\ \chi & \text { generalized exponent, equation (5) [-] } \\ \Phi & \text { Grüneisen parameter, equation (5) [-] } \\ \Psi^{*} & \text { critical flow function, equation (5) [-] }\end{array}$

\section{Indices}

o stagnation state

* $\quad$ critical flow $(M a=1)$

exp experimental data

$s \quad$ isentropic

$T \quad$ isothermal

$v \quad$ isochoric

\section{References}

Annon, DuPont ${ }^{\mathrm{TM}}$, Suva refrigerants, www2.dupont.com/Refrigerants/en_US/products/Suva/i ndex.html.

Annon, 1997, Sonic Nozzles for Mass Flow Measurement and Reference Nozzles for Thrust Verification, AGARD-AR- 321, ISBN 92-836-1056-3.

Bober W., and Chow W.L., 1977, Nonideal Isentropic Flow Through Converging-Diverging Nozzles, ASME J. Fluids Eng., 112(4), pp. 455 -460.

Geropp, D., 1971, Laminare Grenzschichten in ebenen und Rotationssymmetrischen Lavalduesen, Deutsche Luftund Raumfahrt Forschungsbericht, pp.71-90.

Górski, J., 1997, Modeling of Real Gas Properties and its Thermal-Flow Processes, (in Polish). Rzeszow, Oficyna Wyd. PRz.

Johnson, R.C., 1971, Real Gas Effects in the Flow of Methane and Natural Gas Through Critical Flow Nozzles. NASA-TM-X-52994.

Johnson, A.N., 2000, Numerical Characteri-zation of the Discharge Coefficient in Critical Nozzles, Ph.D. Dissertation, Pennsylvania State University, College Station, PA.

Leung J.C., and Epstein M., 1988, A Generalized Critical Flow Model for Nonideal Gases, AIChE J., 34 (9), pp. 1568-1572.

Rabczak, S., 2007, Thermal Equations of State in the Flow Analysis of New Refrigerants, (in Polish), PhD Dissertation, Faculty of Environmental Engineering, Warsaw University of Technology.

Shumann, S.P., 1990, Real Gas Critical Flow Factors for R12, R22 and R502, ASHRAE Transactions, 96(2), pp. 329-336.

Span,R., 2000, Multiparameter Equations of State: An Accurate Source of Thermodynamic Property Data. Springer Verlag, Berlin.

Standard ISO 9300:2005, Measurement of Gas Flow by Means of Critical Flow Venturi Nozzles.

Thompson P.A., and Sullivan D.A., 1977, Simple Predictions for the Sonic Conditions in a Real Gas, ASME Jour. Fluids Eng.; 99(1), pp. 217-225. 